\title{
We're Not Sure We Like It but We Still Want More: Trainee and Faculty Perceptions of Remote Learning During the COVID-19 Pandemic
}

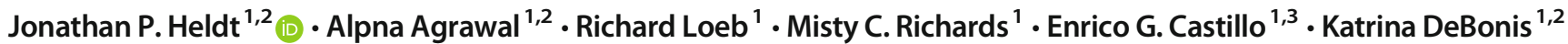

Received: 4 September 2020 / Accepted: 12 January 2021 / Published online: 17 February 2021

(C) This is a U.S. government work and not under copyright protection in the U.S.; foreign copyright protection may apply 2021

\begin{abstract}
Objective In this study, the authors aim to compare perceptions of remote learning versus in-person learning among faculty and trainees at a single institution during the COVID-19 pandemic and to evaluate the impact that a brief faculty training on best practices in online teaching would have on faculty attitudes towards remote learning.

Methods The authors conducted an attitude survey on remote learning among trainees and faculty members approximately 3 months after the transition from in-person to remote learning. The authors then conducted a faculty training on best practices in online teaching followed by an evaluation survey. Study findings were examined descriptively and by Fisher's exact testing.

Results The response rates for the attitudes survey were $68 \%$ among trainees and $61 \%$ among faculty. Trainees and faculty perceived in-person learning more favorably than remote learning across a variety of domains, including overall enjoyment, interpersonal connection, ability to communicate, and concentration. Despite these trends, only $10 \%$ of trainees and $14 \%$ of faculty felt that all lectures would be most effectively delivered in-person when this becomes possible again. The response rate for the faculty training evaluation survey was $16 \%$. Compared to non-attendees, faculty attendees reported more confidence in their ability to teach remotely ( $89 \%$ vs $56 \%, p=0.02$ ) but not increased optimism ( $89 \%$ vs $63 \%, p=0.06)$.

Conclusions The study findings suggest that both trainees and faculty perceive remote learning negatively compared to in-person learning but still feel that some lectures should be delivered remotely even after a return to in-person learning is possible.
\end{abstract}

Keywords Online Education · Graduate Medical Education · COVID-19 pandemic · Educational Technology · Teacher Training

The impact of the COVID-19 pandemic on medical education has been profound. Within undergraduate medical education, the need for social distancing led to the cancellation of many forms of learning including lectures and clerkships [1,2]. Similar steps were taken in graduate medical education, with many trainees experiencing disruptions such as reduced patient volume and abrupt schedule changes [3-5].

Social distancing requirements forced many programs to make major changes to the structure of didactic learning as

Jonathan P. Heldt

jheldt@mednet.ucla.edu

1 University of California, Los Angeles, CA, USA

2 US Department of Veterans Affairs Greater Los Angeles Healthcare System, Los Angeles, CA, USA

3 Los Angeles County Department of Mental Health, Los Angeles, CA, USA well [6]. Anecdotal evidence suggests that many programs transitioned away from in-person learning to remote learning, including both synchronous (livestreamed) and asynchronous (prerecorded) forms [7].

Remote learning is not new, with the majority of residency programs using it at least some of the time [8]. What is novel about the COVID-19 pandemic, however, is the extent of remote learning use and the speed at which it was adopted. The urgency of the situation forced many institutions to implement remote learning within the span of a single week despite the existence of multiple longstanding barriers to adoption and without the opportunity to orient either instructors or learners to the new format [9].

While COVID-19 has restricted in-person gatherings for the time being, we hope that there will come a time when inperson learning becomes both safe and permissible again. For this reason, in this study, we aim to assess the perceptions of remote learning compared to in-person learning among both 
trainees and faculty in our department in order to inform recommendations for structuring didactic experiences at our institution and others. We also aim to assess the possible effects that a brief faculty training focused on best practices in online teaching would have on faculty perceptions of remote learning.

\section{Methods}

In response to the COVID-19 pandemic, all in-person didactics for trainees at our institution were suspended in March 2020, and the curriculum was transitioned to remote learning via the Zoom videoconferencing platform. Following this transition, informal discussions among trainees and faculty indicated that remote learning presented a range of challenges for both learners and teachers.

The purpose of the current study was to understand and address the challenges encountered during the transition from inperson to remote learning among trainees and faculty. First, we conducted a remote learning attitudes survey. The attitudes survey assessed trainees' and faculty's experiences with remote learning compared to in-person learning across domains such as engagement, connection, and convenience using Likert response scales. The survey was distributed to trainees and faculty approximately 3 months after the transition from in-person to remote learning. Eligible participants were all trainees in the adult psychiatry residency $(n=59)$ and child psychiatry fellowship $(n=14)$ as well as all faculty who had taught at least one lecture in the curriculum in the preceding academic year $(n=116)$.

Second, we conducted a 1-h faculty training on best practices in online teaching. The training included three key components: presentation of data from the remote learning attitudes survey, a review of the literature on the efficacy of remote learning, and an introduction to specific remote teaching tools. During this session, we disseminated the following best practice principles for online teaching:

- Understand the literature on remote learning. Many faculty members perceive remote learning to be less effective than in-person learning [10]. Educating faculty on the literature supporting the efficacy of remote learning may help to improve attitudes towards its use.

- Implement active learning. Active learning is an effective method of keeping learners engaged and is associated with increased knowledge retention [11-13]. We introduced several active learning resources (e.g., PollEverywhere and Kahoot) and demonstrated their use within the session itself.

- Encourage learners to turn their cameras on. We encouraged faculty to request that all trainees turn their cameras on at the beginning of each lecture to help with engagement.
- Use trust generators. Trust generators are specific techniques that educators can use to foster a sense of trust between teachers and learners [14]. Given that online platform such as Zoom may be associated with challenges in interpersonal connection [15], the use of trust generators such as selective vulnerability, similarity of interests, and showing concern can be beneficial.

- Use storytelling as a medium. Because listening to a story is an imaginative act, storytelling may survive the transition to remote learning better than other forms of teaching. This is consistent with data supporting the use of storytelling as a method of changing beliefs, attitudes, and behaviors [16].

- Think of remote learning as its own medium with inherent weaknesses and strengths. Finally, we found it helpful to acknowledge that remote learning platforms such as Zoom are inherently different than in-person lectures. In areas where remote learning platforms are less intuitive than in-person teaching, specific features (such as the whiteboard function and breakout rooms in Zoom) may help to bridge the gap. In other areas, remote learning platforms provide features such as the chat function that are not available with in-person teaching.

To assess the impact of the faculty training session, a separate evaluation survey was distributed to all faculty in our department ( $n=334$ ) in July 2020 approximately 2 weeks after the training. Items related to faculty members' knowledge, attitudes, and skills towards remote learning were measured by Likert response scales. Respondents were dichotomized into two cohorts: those who attended the training (attendees) and those who did not (non-attendees).

Survey items for both the attitudes survey and the training evaluation survey were developed by program leadership and trainees knowledgeable about the program's educational curriculum. The protocol for all surveys was IRB exempt (IRB\#20-001287), and data collection was anonymous.

For the remote learning attitudes survey, responses are presented as proportions by trainee and faculty cohorts and discussed descriptively. For the faculty training evaluation survey, responses are dichotomized, and Fisher's exact testing was performed comparing responses from faculty who did and did not attend the training. $P$ values are two-sided with significance set at 0.05 , and Stata 16.1 was used for analyses.

\section{Results}

For the remote learning attitudes survey, the response rate among trainees was $68 \%$ ( $n=50 / 73$ : adult psychiatry residents, $n=41 / 59$ and child psychiatry fellows, $n=9 / 14)$, with $66 \%$ $(n=48 / 73)$ completing the entire survey. Response rates among trainees were similar by training year. Among faculty, the response rate was $61 \%(n=71 / 116$ : adult division, $n=39$, 
child division, $n=20$, and other departments such as the Geriatric Division, Psychology Department, and Volunteer Clinical Faculty, $n=12)$, with $53 \%$ of faculty $(62 / 116)$ completing the entire survey. Faculty respondents represented a range of experience, with $46 \%$ having finished training more than 10 years ago and 54\% less than 10 years ago. For the faculty training evaluation survey, the response rate was $16 \%$ (55/334: attendees, $n=19$ and non-attendees, $n=36$ ).

Trainees and faculty perceived in-person learning more positively than remote learning on the majority of items assessed (Table 1). For example, over half of trainees and faculty indicated overall enjoyment, concentration during lectures, connection between trainees and teachers, and participation in discussions was perceived more positively with in-person lectures than remote lectures. In contrast, trainees and faculty stated remote learning was more convenient compared to in-person learning. While $64 \%$ of faculty felt "much more engaged" seeing learners' faces during remote learning, only $2 \%$ of trainees reported "always" keeping their cameras on while attending remote lectures. Over a third of trainees reported "frequent" $(n=13)$ and "constant" $(n=4)$ distractions during remote lectures. Despite negative attitudes towards remote learning compared to in-person, only a minority of trainees (10\%) and faculty (14\%) felt that all lectures would be most effectively delivered in-person once this becomes safe and permissible.

Among faculty who attended the training session on remote teaching, previous knowledge about specific techniques for improving remote learning was low. Prior to the training, attendees indicated that they had "never heard" of teaching techniques such as trust generators $(21 \%)$, clicker response systems (5\%), real-time collaboration on a document (16\%), the Zoom white board feature (32\%), and Zoom breakout rooms $(5 \%)$. Among non-attendees, many faculty indicated they "did not know" of remote teaching techniques such as trust generators (50\%), storytelling (8\%), clicker response systems (14\%), breakout rooms ( $8 \%$ ), real-time collaboration on a document (6\%), and the Zoom whiteboard function (22\%).

Faculty who attended the training felt more confident in their ability to teach remotely compared to those who did not attend ( $89 \%$ vs $56 \%, p=0.02$ ). Feelings of optimism about teaching remotely did not differ between attendees and nonattendees ( $89 \%$ vs $63 \%, p=0.06$ ). Compared to non-attendees, faculty attendees were more likely to state they would utilize trust generators ( $90 \%$ vs $25 \%, p<0.01)$ and storytelling $(94 \%$ vs $69 \%, p=0.04)$ in future teaching sessions. No significant differences were observed between faculty attendees and nonattendees in their reported likelihood of using clicker systems, real-time collaboration on a document, the Zoom whiteboard function, Zoom breakout rooms, and advance rehearsal of the lecture (Table 2).

\section{Discussion}

Findings from the remote learning attitudes survey indicate that both trainees and faculty prefer in-person learning to remote learning on a majority of domains during the initial period of the COVID-19 pandemic. Despite this, only a minority of trainees and faculty feel that a complete return to in-person learning would be the most effective option when this becomes possible. Given that convenience is the only item that respondents rated as being perceived more positively with remote learning than in-person learning, this suggests that convenience is more important to both trainees and faculty than many other factors. Future studies will be needed to

Table 1 Trainee and faculty preferences for different learning formats

\begin{tabular}{|c|c|c|c|c|c|c|}
\hline & \multicolumn{3}{|c|}{ Trainees $(n=50), \%$} & \multicolumn{3}{|c|}{ Faculty $(\mathrm{n}=71), \%$} \\
\hline & $\mathrm{IP}$ & $\mathrm{R}$ & ND & $\mathrm{IP}$ & $\mathrm{R}$ & ND \\
\hline Overall experience of attending/giving lecture & 50 & 12 & 38 & 84 & 7 & 10 \\
\hline Likelihood of attending/giving lectures & 40 & 42 & 18 & 30 & 13 & 57 \\
\hline Willingness to participate in discussion & 64 & 6 & 30 & 53 & 2 & 46 \\
\hline Willingness to ask questions of learners & $\mathrm{n} / \mathrm{a}$ & $\mathrm{n} / \mathrm{a}$ & $\mathrm{n} / \mathrm{a}$ & 67 & 3 & 30 \\
\hline Enjoyment of lecture & 61 & 18 & 20 & 82 & 10 & 8 \\
\hline Ability to concentrate & 66 & 16 & 18 & 59 & 10 & 31 \\
\hline Ability to communicate material & $\mathrm{n} / \mathrm{a}$ & $\mathrm{n} / \mathrm{a}$ & $\mathrm{n} / \mathrm{a}$ & 85 & 3 & 12 \\
\hline Comprehension of material & 43 & 16 & 41 & $\mathrm{n} / \mathrm{a}$ & $\mathrm{n} / \mathrm{a}$ & $\mathrm{n} / \mathrm{a}$ \\
\hline Retention of material & 49 & 13 & 38 & $\mathrm{n} / \mathrm{a}$ & $\mathrm{n} / \mathrm{a}$ & $\mathrm{n} / \mathrm{a}$ \\
\hline Connection between trainees and instructor & 83 & 6 & 10 & 97 & 0 & 3 \\
\hline Connection between trainees and each other & 74 & 22 & 4 & $\mathrm{n} / \mathrm{a}$ & $\mathrm{n} / \mathrm{a}$ & $\mathrm{n} / \mathrm{a}$ \\
\hline Convenience of coming to lecture & 0 & 90 & 10 & 10 & 78 & 12 \\
\hline
\end{tabular}

$I P$ better in-person, $R$ better remote, $N D$ no difference 
Table 2 Faculty attitudes towards remote teaching and likelihood of using specific remote teaching techniques

\begin{tabular}{|c|c|c|c|}
\hline & Attendees $(n=19), \%$ & Non-attendees $(n=36), \%$ & $\mathbf{p}$ \\
\hline \multicolumn{4}{|l|}{ Attitudes towards remote teaching } \\
\hline Confidence & 89 & 56 & 0.02 \\
\hline Optimism & 89 & 63 & 0.06 \\
\hline \multicolumn{4}{|c|}{ Likelihood of using specific remote teaching techniques' } \\
\hline Trust generators & 90 & 25 & $<0.01$ \\
\hline Storytelling & 94 & 69 & 0.04 \\
\hline Clicker systems & 63 & 39 & 0.08 \\
\hline Real-time collaboration on a document & 47 & 44 & 0.53 \\
\hline Zoom whiteboard function & 32 & 31 & 0.59 \\
\hline Zoom breakout rooms & 63 & 50 & 0.26 \\
\hline Advance rehearsal of the lecture & 68 & 78 & 0.33 \\
\hline
\end{tabular}

evaluate this further, as we did not ask survey respondents to assign relative weights to each item.

There is discrepancy between trainees and faculty regarding the perceived efficacy of remote learning. While $85 \%$ of faculty indicated that communication of material was perceived more positively with in-person learning compared to remote, over half of trainees perceived that comprehension and retention of material were either no different or better with remote learning. These data may be reassuring to faculty. Trainee perceptions are also more in line with the preCOVID-19 literature which is generally positive towards remote learning [17]. This provides additional evidence for a gap between perceptions of remote learning and its actual efficacy in teaching, a gap which has previously been reported in the literature [18-20].

It is possible that the rapid speed of adoption may have had an adverse effect on perceptions of remote learning, as COVID-19 forced all faculty in our department to engage in remote teaching regardless of prior experience or training. To examine the effect that additional training could have on faculty, we presented a 1-h training focused on best practices in online teaching. Faculty who attended this brief training reporting feeling more confident in their ability to teach remotely compared to faculty who did not attend the training. However, it is less clear that this cohort is more likely to use the specific techniques that were discussed, with only two techniques (trust generators and storytelling) being more likely to be used by attendees of the training compared to non-attendees. Both of these techniques are easily understandable, require little technical ability, and may feel intuitive to mental health professionals. In contrast, more technologically advanced methods such as clicker systems, real-time collaboration on a document, and breakout rooms were not associated with increased use after the training session. We believe this is because many faculty members had little direct experience with these technologies, and the training did not include stepwise instructions on how to use them. A single hour of training, while sufficient for changing attitudes about remote learning, may not be enough to change practices. For this reason, additional training focused on how to use a specific types of educational technology may be warranted.

Ultimately, our study must be viewed in light of its limitations. Our data reflect perceptions at a single institution, raising concerns about generalizability. As our institution adopted primarily synchronous remote learning, it is possible that other departments who use asynchronous remote learning may have different experiences. There is also a risk of selection bias, as our response rates were between 50 and $65 \%$ for the attitudes survey and lower for the faculty training evaluation survey.

In conclusion, our data provide preliminary evidence that, while remote learning is perceived less favorably than in-person learning by a majority of trainees and faculty alike in the initial period of the COVID-19 pandemic, most trainees and faculty felt that it would be most effective for remote learning to continue in some form even after in-person learning becomes possible. Our data also suggest that a brief faculty training can improve attitudes towards remote learning, although additional training may be needed to impact specific behaviors. It is worth highlighting that our study specifically assessed attitudes towards various forms of learning but did not directly involve more objective learning outcomes such as trainee performance on board examinations and the PRITE, which could be a direction for future study. We hope that additional research will be conducted to provide guidance to training programs desiring to maintain a high quality of didactic education not only in the era of COVID-19 but in the future as well.

\section{Compliance with ethical standards}

Disclosure The authors declare no conflict of interest. 


\section{References}

1. Akers A, Blough C, Iyer MS. COVID-19 implications on clinical clerkships and the residency application process for medical students. Cureus. 2020;12(4):e7800.

2. Ferrel MN, Ryan JJ. The impact of COVID-19 on medical education. Cureus. 2020;12(3):e7492.

3. Chick RC, Clifton GT, Peace KM, Propper BW, Hale DF, Alseidi AA, et al. Using technology to maintain the education of residents during the COVID-19 pandemic. J Surg Educ. 2020;77(4):729-32.

4. Chiel L, Winthrop Z, Winn AS. The COVID-19 pandemic and pediatric graduate medical education. Pediatrics. 2020;146(2): e20201057. https://doi.org/10.1542/peds.2020-1057.

5. Porpiglia F, Checcucci E, Amparore D, Verri P, Campi R, Claps F, et al. Slowdown of urology residents' learning curve during the COVID-19 emergency. BJU Int. 2020;125(6):E15-7.

6. Gottlieb M, Landry A, Egan DJ, Shappell E, Bailitz J, Horowitz R, et al. Rethinking residency conferences in the era of COVID-19. AEM Educ Train. 2020;4(3):313-7.

7. Parslow GR. Commentary: synchronous and asynchronous learning. Biochem Mol Biol Educ. 2012;40(3):212.

8. Wittich CM, Agrawal A, Cook DA, Halvorsen AJ, Mandrekar JN, Chaudhry S, et al. E-learning in graduate medical education: survey of residency program directors. BMC Med Educ. 2017;17(1):114.

9. O'Doherty D, Dromey M, Lougheed J, Hannigan A, Last J, McGrath D. Barriers and solutions to online learning in medical education - an integrative review. BMC Med Educ. 2018;18(1):130

10. Pomerantz J, Brooks DC. ECAR study of faculty and information technology, 2017. Louisville: ECAR; 2017.

11. Freeman S, Eddy SL, McDonough M, Smith MK, Okoroafor N, Jordt $\mathrm{H}$, et al. Active learning increases student performance in science, engineering, and mathematics. Proc Natl Acad Sci U S A. $2014 ; 111(23): 8410-5$.
12. Ballen CJ, Wieman C, Salehi S, Searle JB, Zamudio KR. Enhancing diversity in undergraduate science: self-efficacy drives performance gains with active learning. CBE Life Sci Educ. 2017;16(4):ar56.

13. Priego-Quesada JI, Jimenez-Perez I. Cibrian Ortiz de Anda RM, Gonzalez-Pena R, Salvador Palmer R. Effect of in-class group clicker-quiz competition on student final exam performance. Adv Physiol Educ. 2019;43(3):430-4.

14. Hammond Z, Jackson Y. Culturally responsive teaching and the brain: promoting authentic engagement and rigor among culturally and linguistically diverse students. Corwin, a SAGE company: Thousand Oaks; 2015.

15. Cooley L. Fostering human connection in the COVID-19 virtual health care realm. NEJM Catal. 2020. https://doi.org/10.1056/CAT. 20.0166 .

16. Braddock K, Dillard JP. Meta-analytic evidence for the persuasive effect of narratives on beliefs, attitudes, intentions, and behaviors. Commun Monogr. 2016;83(4):446-67.

17. Pei $\mathrm{L}, \mathrm{Wu} \mathrm{H}$. Does online learning work better than offline learning in undergraduate medical education? A systematic review and meta-analysis. Med Educ Online. 2019;24(1):1666538.

18. Moridani M. Asynchronous video streaming vs. synchronous videoconferencing for teaching a pharmacogenetic pharmacotherapy course. Am J Pharm Educ. 2007;71(1):16.

19. Kunin M, Julliard KN, Rodriguez TE. Comparing face-to-face, synchronous, and asynchronous learning: postgraduate dental resident preferences. J Dent Educ. 2014;78(6):856-66.

20. Lew EK, Nordquist EK. Asynchronous learning: student utilization out of sync with their preference. Med Educ Online. 2016;21:30587.

Publisher's Note Springer Nature remains neutral with regard to jurisdictional claims in published maps and institutional affiliations. 